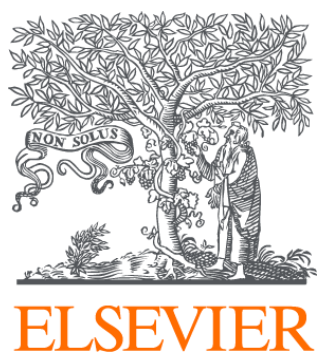

Since January 2020 Elsevier has created a COVID-19 resource centre with free information in English and Mandarin on the novel coronavirus COVID-

19. The COVID-19 resource centre is hosted on Elsevier Connect, the company's public news and information website.

Elsevier hereby grants permission to make all its COVID-19-related research that is available on the COVID-19 resource centre - including this research content - immediately available in PubMed Central and other publicly funded repositories, such as the WHO COVID database with rights for unrestricted research re-use and analyses in any form or by any means with acknowledgement of the original source. These permissions are granted for free by Elsevier for as long as the COVID-19 resource centre remains active. 


\title{
The TYMV tRNA-like structure
}

\author{
R Giegéa ${ }^{a}$, C Florentz ${ }^{a}$, TW Dreher ${ }^{b}$ \\ aUnité 'Structure des Macromolécules Biologiques et Mécanismes de Reconnaissance', \\ Institut de Biologie Moléculaire et Cellulaire du Centre National de la Recherche Scientifique, \\ 15 rue René Descartes, F-67084 Strasbourg Cedex, France; \\ bOregon State University, Department of Agriculture and Life Sciences, Corvallis, OR 97331-6502, USA
}

(Received 19 January 1993; accepted 18 February 1993)

\begin{abstract}
Summary - The genomic RNA from turnip yellow mosaic virus presents a 3'-end functionally and structurally related to tRNAs. This report summarizes our knowledge about the peculiar structure of the tRNA-like domain and its interaction with tRNA specific proteins, like RNAse P, tRNA nucleotidyl-transferase, aminoacyl-tRNA synthetases, and elongation factors. It discusses also the biological role of this structure in the viral life cycle. A brief survey of our knowledge of other tRNA mimicries in biological systems, as well as their relevance for understanding canonical tRNA, will also be presented.
\end{abstract}

turnip yellow mosaic virus RNA / tRNA-like structure / aminoacylation / replication

\section{Introduction}

Turnip yellow mosaic virus, the type member of the tymoviruses and causative agent of systemic infections primarily in members of the Cruciferaea, was discovered in the late 40s [1] and since then has been studied extensively (reviewed by Hirth and Givord [2]). This spherical virus has a characteristic icosahedral capsid, formed by 180 copies of the coat protein monomer $(20 \mathrm{kDa})$. The capsid contains one copy of a single stranded $(+)$ sense genomic RNA. This RNA (6318 nucleotides) has been sequenced for two different strains, the type strain originally from Strasbourg $[3,4]$ and an Australian isolate [5]. It contains two overlapping 5'-proximal genes and a silent 3'-proximal coat protein gene. In infected host cells, a subgenomic RNA of 693 nucleotides is generated and is also encapsidated; its sequence codes for the coat protein. The two 5'-proximal genes are translated from the genomic RNA. A long open reading frame encodes a

Abbreviations: TYMV, turnip yellow mosaic virus; BMV, brome mosaic virus; TMV, tobacco mosaic virus; TYMC, Corvallis strain of TYMV RNA; TY-Alu, TY-Dde, TY-Dra, TYSma, clones of cDNA fragments of different length starting at restriction sites Alu, Dde, Dra, Sma and containing the tRNAlike domain of TYMV RNA; TY-AA, clone of cDNA containing the amino acid accepting branch of TYMV RNA; aaRS, aminoacyl-tRNA synthetase (amino acids are abbreviated by the three-letter code); $\mathrm{CP}$, coat protein; ORF, open reading frame.
206-kDa protein that undergoes specific cleavage resulting in two proteins of about 150 and $70 \mathrm{kDa}[6$, 7]. A second open reading frame, upstream of the previous one and out of frame, encodes a $69-\mathrm{kDa}$ protein [8]. A cDNA clone from which infectious transcripts of the genomic RNA can be made in vitro has been constructed, and has opened the way to genetic analysis of the viral genome in connection with the viral life cycle [8].

TYMV was the first virus for which a very peculiar feature was discovered, namely the presence of a tRNA-like domain at the 3'-end of its RNA. The first experimental suggestions pointing to a functional tRNA mimicry were reported about 30 years ago when it was suggested that the viral RNA can bind amino acids, predominantly valine, when incubated with bacterial extracts and that this binding is $\mathrm{pH}$ dependent and lost at alkaline $\mathrm{pH}$ [9]. This last fact is reminiscent of the properties of the ester bond between amino acids and the terminal ribose of tRNAs. Firm démonstration that valine dan be attached by a covalent bond to the 3'-extremity of the viral RNA by a reaction catalyzed by ValRS was presented by Pinck et al [10] and Yot et al [11]. The immediate consequence of this discovery was the expectation that the viral RNA contained a structure mimicking a tRNA. Soon afterwards, it was also discovered that this tRNA mimicry is not restricted to aminoacylation, but concerns interaction of the viral RNA with other tRNA-specific proteins like elongation factors [12, 13], tRNA nucleotidyl-transferase [14], and RNAse P 
A

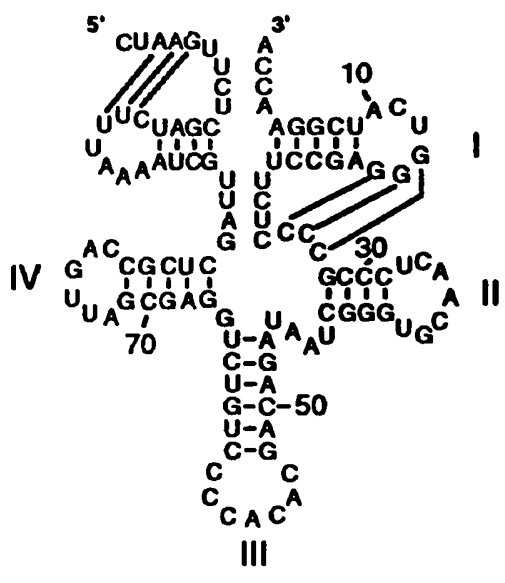

B

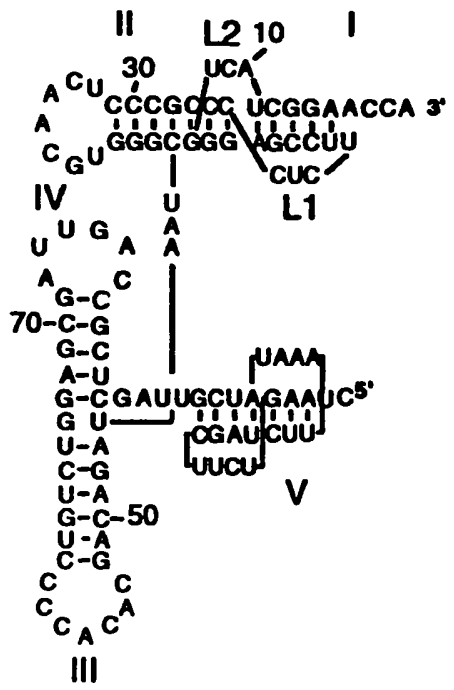

C

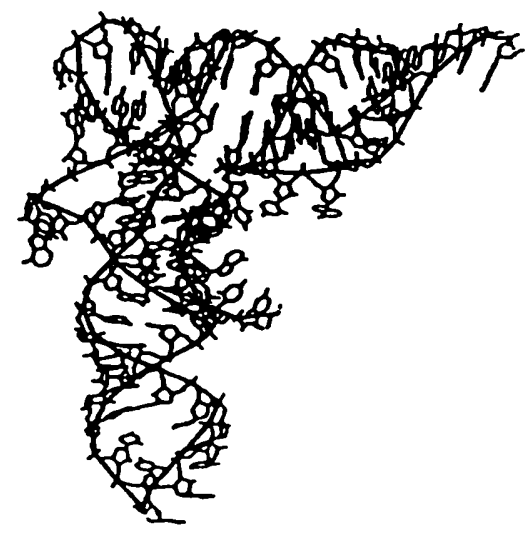

Fig 1. Folding of the 3'-end of TYMV RNA. A. Secondary structure of the last 110 nucleotides of the viral RNA, including the IRNA-like domain. Numbering starts at the 3'-end with negative numbers (for simplicity the minus signs are omitted). Plain lines highlight long range tertiary interactions, leading to the formation of pseudoknots. B. Secondary structure highlighting the pseudoknots and the general L-shaped conformation. C. Graphical three-dimensional computer model of the $863^{\prime}$-nucleotides [27]. In B, residue U21 from loop L1 of the pseudoknot is placed opposite to discriminator base A4.

[15]. The functional tRNA mimicry is however not complete, since TYMV RNA is not amino acid donor in protein synthesis [16].

In this paper we summarize the present view on the structure and function of the IRNA-like domain of TYMV RNA. We describe the mimicry of this structure for tRNA recognizing enzymes (RNAse P, tRNA nucleotidyl-transferase, aminoacyl-tRNA synthetases, and elongation factor) and discuss its role in the viral life cycle. We show in particular its involvement in the replication of the viral genome. Finally we summarize our knowledge on the tRNA mimicries in other plant viral RNAs and show that such mimicries are widespread events.

\section{Structure of the 3 '-non-coding region of TYMV RNA}

Because TYMV RNA could be valylated by ValRS with kinetic characteristics similar to those of the charging of canonical tRNA [17], it could be anticipated that the viral RNA contains structural features resembling those of tRNA val for the recognition and charging by the synthetase. The surprise was great that no obvious resemblance between a tRNA cloverleaf and the sequence of the 3'-end of the viral RNA was found $[18,19]$. Thus the mimicry had to be searched for at the conformational level of the molecule. In what follows we describe the structural studies, mainly biochemical, leading to the present structural knowledge of the TYMV tRNA-like domain. It is worth emphasizing the importance these studies had for understanding RNA, because they led to the discovery of a new folding principle in these molecules, the pseudoknot, which is one of the major recent discoveries in the RNA field [20, 21]. Indeed, pseudoknots appear widespread in nature and are found to be involved in an increasing number of biological processes (reviewed by ten Dam et al [22] and Westhof and Jaeger [23]).

\section{Secondary structure}

RNA fragments of 112 and 159 nucleotides, corresponding to TYMV RNA have been purified from partial RNAse digests of the viral RNA, and their secondary structure determined experimentally and independently by two groups using enzymatic and chemical probing techniques [24-26]. Both approaches lead to the same folding. The longest of these fragments, containing the end of the coat protein gene, was shown to be as efficient a substrate for ValRS as the complete viral RNA [24] suggesting that this fragment contains all the chemical information required for recognition by the aminoacyl-tRNA synthetase. Figure $1 \mathrm{~A}$ represents the secondary structure of the noncoding 110 last nucleotides including the stop codon for the coat protein cistron at position -107 to -109 . 
The secondary structure of the 1103 '-terminal nucleotides of TYMV RNA is divided in five wellorganized stem and loop regions. Three of them (II, III, IV) present strong analogies in respect to their size with the T-, anticodon-, and D-stems and loops of canonical tRNAs. Two single stranded regions equivalent to the variable loop and to the conserved CCA 3 -terminal sequence are also present. Conversely, hairpins I and $V$ have no analogous counterpart in classical tRNAs. This secondary structure of the TYMV tRNA-like domain is supported by phylogenetic data, since five other valylatable tymoviral tRNA-like domains and one tobamoviral valylatable RNA fold similarly over their last 80 nucleotides [28].

\section{Three-dimensional fold}

Interpretation of experimental data on the reactivity of some nucleotides of loop I and of the single-stranded region between hairpins I and II to nucleases, and the observation of compensatory base changes in different valylatable RNA strains, brought Pleij and his coworkers to propose the presence of long-range interactions [26]. These interactions lead to the formation of a pseudoknot at the 3'-end of the RNA (fig IB) and contribute to the formation of an amino acid acceptor arm very similar to the one found in classical tRNAs. Indeed, the stacking of three helical domains, namely stem II, the three base-pairs formed by the tertiary interactions, and stem 1 , leads to a 12 base-pair-long helix. As in tRNAs, the four last nucleotides of the 3 '-end remain free. Two short single-stranded stretches ( $\mathrm{Ll}$ and L2) comprising three and four nucleotides, respectively, cross the helix from one strand to the other and ensure the chain continuity. The reality of this pseudoknot, and thus of the folding of the acceptor arm, is supported by several additional arguments. Firstly, similar long-range interactions exist in the other sequenced tymoviral tRNA-like domains and in the RNA of the valine accepting strain of TMV [28]. Secondly, the necessity of the presence of the pseudoknot for aminoacylation has been assessed by a careful mutational analysis; any mutation disrupting the pseudoknot is harmful to aminoacylation [29].

A second pseudoknot can be formed at the 5'-end of the non-coding region of TYMV RNA (fig IA, B). The presence of this pseudoknot was suggested by Rietveld [30] and arguments in favour of the involvement of loop $\mathrm{V}$ in intramolecular interactions were presented by Florentz and Giegé [31]. Genetic support for the existence and functional importance of this second pseudoknot was obtained recently (see below).

A three-dimensional model of the 3' 82 nucleotides has been built by computer modelling on a graphics system (fig 1C) [27]. This was the first computer modelling of an RNA molecule, a field which has since then developed rapidly [32] because of the difficulty in crystallizing RNA molecules [33, 34]. The amino acid acceptor arm portion of the model was constructed de novo. It shows that a pseudoknot can form according to the stereochemical rules known for RNAs. The rest of the molecule was constructed on the basis of the crystallographically solved structure of yeast tRNAAsp [35] and gives a general view of the L-shaped conformation of thie tRNA-like region. This conformation mimics perfectly the fold of a classical tRNA, especially as far as the relative positioning of the amino acid end and the anticodon region is concerned. However, the network of tertiary interactions in the core of the molecule, although not determined with certainty, differs from that found in tRNA. This is also the case for the link of the remaining viral genome with the tRNA-like domain, which is different from that of the extra-RNA domains present in precursor tRNAs. While the viral RNA chain leaves the tRNA-like domain in the hinge region of the L-shaped domain [36], the extra-nucleotides in precursor tRNAs extend the molecule at their 5'- and sometimes 3'-ends, which are in close vicinity to each other.

\section{Interaction with proteins from the tRNA metabo-} lism and function: in vitro aspects

\section{Interaction with RNAse P}

Ribonucleases $\mathbf{P}$ are involved in the maturation of pretRNAs. These enzymes, composed of a catalytic RNA moiety and a protein subunit, generate mature 5 '-ends of tRNAs (reviewed by Altman [37] and Brown and Pace [38]). The tRNA-like structure from TYMV RNA is substrate for RNAse P from Escherichia coli [39] and Bacillus subtilis [40]. Cleavage occurs at a position stereochemically equivalent to the cleavage site in canonical tRNAs, namely at the end of the amino acid acceptor arm, at the 3'-end of loop Ll. Cleavage does not require the anticodon region of the tRNA, and is dependent upon the presence of a pyrimidine residue at the clevage site. Cleavage does not require the pseudoknot, although the cleavage rate and specificity are increased in the presence of such a special folding [41]. Additionaly, the nature of the nucleotide $5^{\prime}$ to the CCA-terminus, as well as the sequence of the first base-pair of the acceptor arm, are of importance.

\section{Interaction with tRNA nucleotidyl-transferase}

tRNA nucleotidyl-transferases are other enzymes involved in the maturation of tRNAs (reviewed by Deutscher [42]) and are responsibie for the presence of an accurate 3'-end CCA sequence in these mole- 
cules. The enzyme from $E$ coli incorporates a terminal adenosine to the TYMV RNA deprived of the terminal A [14]. Efficient recognition by this enzyme requires about 75 nucleotides [43].

\section{Interaction with aminoacyl-tRNA synthetases}

Valylation properties of the tRNA-like structure The tRNA-like domain of TYMV RNA is efficiently recognized by yeast ValRS. Indeed, the kinetic parameters of valylation of the genomic and the subgenomic RNAs of TYMV are very close to those measured for valylation of the cognate tRNA ${ }^{\text {vil }}$ [17]. The efficiency in aminoacylation of the tRNA-like structure (estimated by the ratio between the kinetic parameters $k_{\mathrm{Cat}}$ and $K_{\mathrm{m}}$ ) is 20-30 times lower than that of the cognate tRNA $v_{\text {itl }}$. This is very efficient when compared to the loss in valylation efficiencies of tRNA ${ }^{\text {Phe }}$ or tRNAMet, the best non-cognate substrates for yeast ValRS [44], which have reduced efficiencies of 4-5 orders of magnitude.
Optimal length for aminoacylation activity Equivalent kinetic parameters of valylation have been measured for the complete genomic RNA and for a fragment of 159 nucleotides containing the end of the coat protein cistron. An unsolved question however, concerns the length of the minimal fragment capable of being aminoacylated. A qualitative answer to this question was obtained by assessing the aminoacylarion abilities of fragments originating from nuclease cleavage of the viral RNA. The shortest aminoacylatable fragment was found to be $82 \pm 2$ nucleotides [43]. Interestingly, such a fragment corresponds to the size required for the formation of the L-shaped structure (sec fig 1). A more precise analysis was based on the measuraments of kinetic parameters of aminoacylation of a series of RNA fragments of different length [45]. In vitro transcripts corresponding to TYMV RNA 3'-end fragments of 88, 99, 115 or 264 nucleotides, respectively, showed that the shortest fragments are incompletely charged and that the aminoacylation rates were slower than the rate observed for
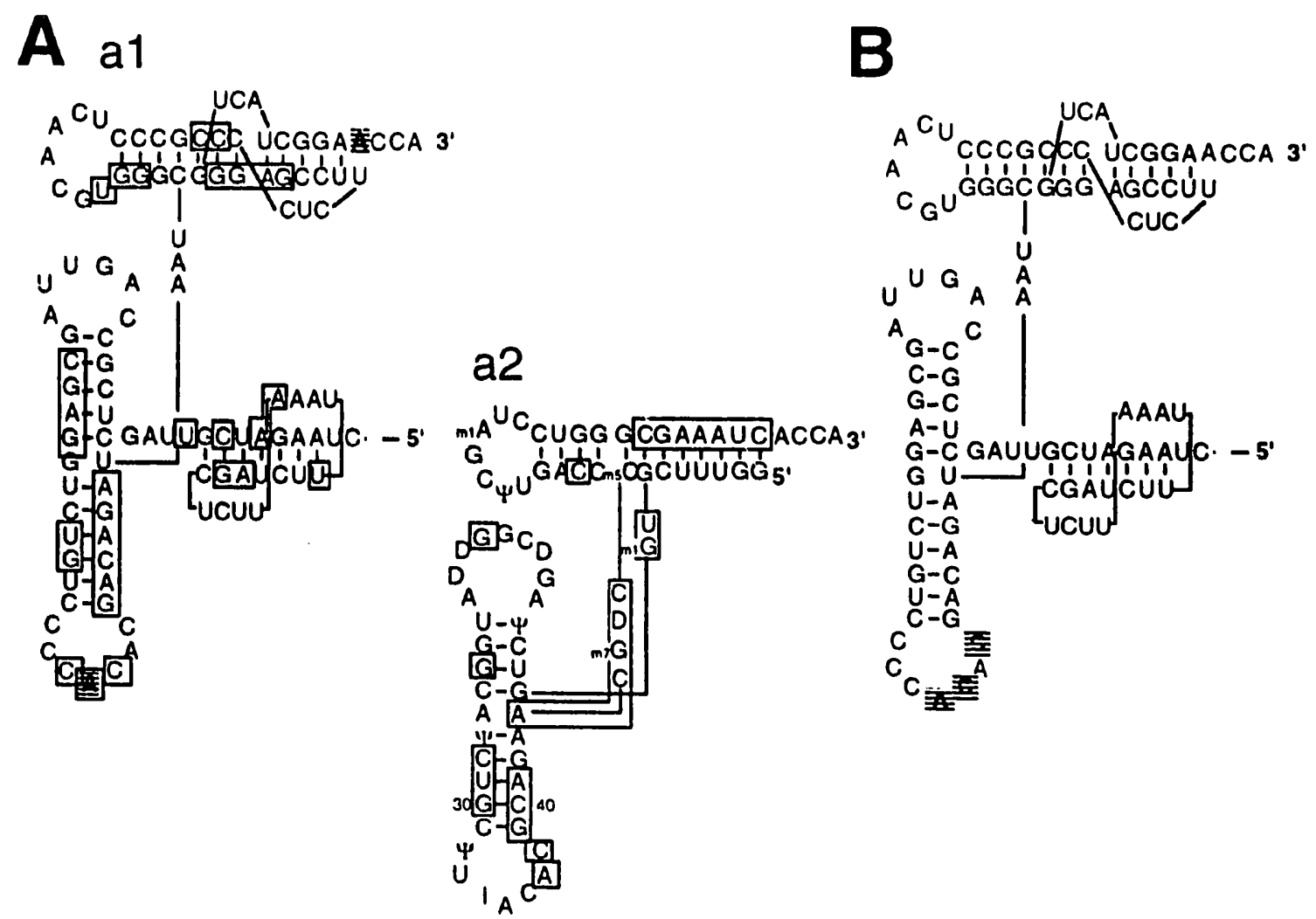

Fig 2. Recognition of the TYMV IRNA-like domain with ValRSs (A, B). A, al. Contact points with yeast ValRS (boxed regions) and identity nucleotides required for efficient aminoacylation by this enzyme (dashed nucleotides). $\mathbf{A}$, a2. Contact points of yeast tRNA vil with its cognate synthetase (data are from [47]). B. Identity nucleotides for efficient aminoacylation by wheat germ ValRS. For pseudoknot residue U21, see legend to figure 1 . 
the longest transcript. Thus it appeared that nucleotides upstream the core region formed by the 3' 82 nucleotides, are important for optimal valylation. As will be shown below, nucleotides of this upstream region interact with yeast ValRS. Another and nonexclusive explanation for the lower efficiency of short fragments concerns their potential to form alternative inactive conformations [46]. Indeed, the kinetic analyses were performed on in vitro transcripts presenting six non-viral nucleotides at their 5'-end [45]. Whereas these extra-nucleotides probably have no influence on the folding of the longest tRNA-like molecule, they may interfere with the folding of the shorter ones and thus hinder efficient aminoacylation [46]. Thus, the sequence of 82 nucleotides forming the L-shaped structure of the tRNA-like domain appears to possess the full potential for optimal aminoacylation, but interaction between ValRS and external 5'-sequences may help to stabilize binding of the tRNA-like domain with the enzyme and consequently facilitate its charging.

\section{Recognition by yeast and wheat germ valyl-tRNA synthetase}

The signals within the tRNA-like domain recognized by ValRS and important for specific aminoacylation have been searched for using two approaches: the contact points with yeast ValRS have been defined by footprinting experiments and the nucleotides important for aminoacylation by yeast and wheat germ ValRS have been defined by the kinetic analysis of a series of mutated tRNA-like fragments, obtained by in vitro transcription.

The natural 159 nucleotide-long tRNA-like fragment is protected by yeast ValRS against digestion by nucleases and against chemical modification of phosphates by enthylnitrosourea essentially in the anticodon and the acceptor arms [31] (fig 2). Interaction with the acceptor arm occurs in spite of the presence of the pseudoknot and shows that the yeast enzyme tolerates some structural variability in this part of its substrate. The global interaction domains are equivalent to the major sites of interaction of ValRS with its cognate tRNA Val. These results were thus further evidence supporting the L-shaped folding proposed for the TYMV tRNA-like domain. Interestingly, yeast ValRS interacts also with the region upstream of the L-shaped part of the tRNA-like structure. This additional interaction may contribute to a better stabilization of the tRNA/synthetase complex [31].

Nucleotides important for valylation have been searched for by a mutational analysis of the tRNAlike domain $[48,49]$. Variants of the tRNA-like structure were created via site-directed mutagenesis of a cDNA clone (covering the 258 last nucleotides of TYMV RNA) and in vitro transcription with T7 RNA polymerase. Two main loci were investigated for their importance in aminoacylation by both yeast and wheat germ enzymes, namely the anticodon loop and the 'discrimirator' base in the acceptor arm (the fourth base starting from the 3'-end). Indeed, sequence comparisons with a consensus sequence of higher eukaryotic tRNA val pointed to strong homologies with the anticodon and its 3 '-flanking bases [28, 50]. Mutation of the central position of the anticodon triplet, replacing A-56 by $G$ or $U$, resulted in a dramatic decrease in the efficiency of valylation by the yeast enzyme (decrease by four orders of magnitude [48]) indicating that this nucleotide is a very strong element, an identity nucleotide, for valylation. Mutation of the anticodon affects the catalysis in a kinetic- rather than affinity-based manner. Indeed, the mutants are still able to interact with the synthetase with affinities comparable to those of the wild-type transcript and behave as competitive inhibitors in the valylation reaction of

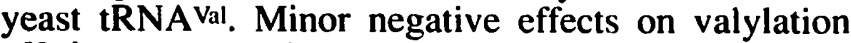
efficiency were observed for mutants with substitutions at the discriminator base, suggesting some contribution to recognition and catalysis by yeast ValRS.

Further studies with wheat germ ValRS investigated in detail the contribution of the anticodon loop nucleotides (see table I) and of the discriminator base to valine identity [49]. Substitution of the discriminator base did not affect valylation, showing the nonimportance of this position for wheat germ ValRS. These results suggest already a different recognition pattern by both types of eukaryotic ValRS. Most individual substitutions in the middle (A-56) or 3' (C-55) positions of the anticodon triplet resulted in very poor valylation $\left(V_{\max } / K_{\mathrm{m}}\right.$ values more than 1000 -fold lower than wild-type); the smallest effect resulted from the C-55U substitution (a 59 -fold decrease in $V_{\text {mix }} / K_{\mathrm{m}}$ ). Nucleotides A-56 and C-55 are thus clearly strong valine identity determinants. A third determinant in the anticodon loop was identified from the study of double mutants. While individual substitutions of C-53 resulted in mild loss of valylation (3- to 11 -fold decreases in $\left.V_{\max } / K_{\mathrm{m}}\right)$, the combined C-55U and C-53A substitutions resulted in drastically reduced valylation (2400-fold decrease in $V_{\text {max }} / K_{\mathrm{m}}$ ). Residue C-53 is thus an important valine identity determinant recognized by wheat germ ValRS, but less potent than those in the anticodon triplet. Mutation of nucleotides A-54 or C-57 yielded RNAs with kinetic parameters not significantly different from those of wild-type RNA. Thus these nucleotides were not considered as valine identity elements. Conversely to what happens with yeast ValRS, the discrimination by wheat germ ValRS appeared to be more based on affinity than on kinetic events, since the valylation of most mutants was mainly affected at the level of $K_{\mathrm{m}}$.

Analysis of the behaviour of multiple mutants within the anticodon loop allowed to define the relationship between the three identity nucleotides. 
Table I. Correlation between valylation and replication of TYMV RNAs.

\begin{tabular}{|c|c|c|c|}
\hline Mutant & $\begin{array}{l}\text { Anticodon loop } \\
\text { sequence }\end{array}$ & $\begin{array}{l}\text { Relative efficiency } \\
\text { of in vitro valylation }\end{array}$ & $\begin{array}{l}\text { Relative replication } \\
\text { in protoplasts }\end{array}$ \\
\hline Wild-type & 59-СCCACAC-53 & 100 & 100 \\
\hline TY-U-57 & . $\underline{\mathrm{U} . . .}$ & 114 & 1.5 \\
\hline TY-G-56 & $\ldots \mathbf{G} \ldots$ & 0.02 & $\leq 0.2$ \\
\hline TY-A-55 & $\ldots$ A & 0.46 & 2.0 \\
\hline TY-C-54 & $\ldots \mathrm{C}$ & 22 & 24 \\
\hline TY-A-53 & $\ldots \ldots A$ & 9.3 & 0.12 \\
\hline TY-U-57/A-55 & . U.A. & 7.5 & 85 \\
\hline
\end{tabular}

In vitro valylation efficiencies $\left(V_{\mathrm{max}}^{\prime} / K_{\mathrm{m}}\right)$ were determined on 264-nucleotide-long 3'-fragments of TYMV RNA using wheat germ ValRS (data from [49]). The replication levels report the accumulations of coat protein in Chinese cabbage protoplasts 48 $\mathrm{h}$ after inoculation (data from [66]). aAnticodon positions are underlined.

Indeed, if the effects are additive, the relative specificity constants (relative $V_{\text {nua }} / K_{\mathrm{m}}$ ) of a double mutant equals the product of the relative specificity constants of the single mutants [51]. In cases of cooperative or anti-cooperative effects, the calculated values of the relative specificity constants will deviate from the experimental determinations. This approach allowed to detect a mild cooperative effect between nucleotides C-53 and C-55. Moreover, they revealed an unexpected contribution of nucleotide A-54. Indeed. multiple mutants involving changes at the level of these nucleotides in combination with changes at nucleotide(s) -55 and/or -57 . had relative $V_{\text {max }} / K_{m}$ 1.9-2.6 times lower than expected if the individual mutations were additive.

Conversely, the double mutant U-57/A-55 is a much better substrate than expected and shows compensatory effects between both nucleotides. This anticooperativity may reflect that ValRS is sensitive to the conformation of the phosphate backbone as well as to functional groups on the bases of the anticodon [49]. Interestingly, this anti-cooperative behaviour was also observed in vivo [52]. Indeed, a TYMV genomic RNA variant, mutated in the tRNA-like domain (mutation C-55 to A) and thus impaired in its aminoacylation and replication capacities, inoculated to plants, became capable of systemic infection after several weeks in host cells. Sequencing of the progeny viral RNAs revealed the occurrence of a compensatory mutation (C-57 to $\mathrm{U}$ ). This is an example. at the molecular level, showing that alternative solutions exist for a same biological recognition problem, likely involving compensatory effects within RNA/protein contacts.
Aminoacylation of TYMV RNA is sensitive to local conformations in the RNA [36]. Mutating residue $\mathrm{U}-43$ to $\mathrm{C}-43$, at the junction of the amino acid accepting and anticodon branches of the tRNAlike domain, drastically impairs the valylation capacity of the variant, presumably because it allows the formation of a new G-83/C-43 base-pair that brings the remaining viral RNA closer to the tRNA-like domain and consequently hinders interaction with ValRS. An extensive study of variants mutated in the pseudoknotted region showed that as long as the pseudoknot can form, there is no dramatic change in the valylation of the tRNA-like fragment. However, if the pseudoknot cannot be formed, valylation is suppressed [29].

\section{Degenerated specificity}

The specificity of tRNA aminoacylation is often not absolute since synthetases can catalyze mischarging reactions (reviewed by Ebel et al [53] and Giegé et al [54]). This possibility was also suggested for the aminoacylation of TYMV RNA after detection of the binding of amino acids other than valine to viral RNA samples by $E$ coli aminoacyl-tRNA synthetases [28]. However, in these experiments it was not excluded that these amino acids were bound to contaminating cellular tRNAs. Charging of the tRNA-like domain with non-cognate amino acids was explicitely demonstrated by aminoacylation studies of in vitro RNA transcripts [55]. Whereas the 254 nucleotide-long TYSma transcript is neither recognized by ArgRS, AspRS nor by PheRS, it is an efficient substrate for yeast HisRS. The efficiency of histidinylation however, is dependent upon the length of the transcript. 
The 88-nucleotide long TY-Alu transcript is the best substrate with only five times lower catalytic efficiency of charging than the corresponding wild-type tRNA ${ }^{\text {His }}$ transcript. Increasing the length of transcripts decreases their ability to be recognized by HisRS, suggesting that the upstream sequences hinder recognition by steric effects. Mischarging occurs not only with wild-type RNA transcripts but also with variants mutated at valine identity positions, thus demonstrating that valine and histidine identities do not overlap, and more specifically, that the middle position of the valine anticodon does not participate in histidine identity.

Histidinylation of TYMV RNA fragments of different length pointed to an efficient histidinylation of a minihelix containing a pseudoknot (fragment TYAA). This minihelix corresponds to the amino acid acceptor arm of the tRNA-like structure, thus convincingly supporting the view that the major nucleotides responsible for histidine specificity are located in the amino acid accepting arm (fig 3). Likely, in this molecule, as in canonical tRNAHis from $E$ coli [56] and in minihelices derived from this tRNA [57], an additional nucleotide at position -1 is present for identity. This nucleotide would be the first nucleotide of loop L 1 from the pseudoknot in the case of the tRNA-like molecule. Interestingly this nucleotide has been shown to be stacked on top of the acceptor arm helix as is residue -1 in tRNAHis $[27,55]$ (fig $1 \mathrm{C}, \mathrm{D})$. Whereas aminoacylation of classical minihelices has been demonstrated in a number of cases [58], studies on the tRNA-like molecule led to the first evidence for the aminoacylation of a pseudoknotted minihelix.

The mischarging of TYMV RNAs and the derivatives mentioned above has also been studied with wheat germ HisRS (Tsai and Dreher, unpublished). Under a variety of conditions, a maximal charging level of $0.08 \mathrm{~mol}$ histidine/mol RNA was observed for RNAs containing the entire tRNA-like structure, and $0.15 \mathrm{~mol} / \mathrm{mol}$ for TY-AA RNA. These results imply that TYMV RNA would only become moderately histidinylated in its higher plant host cells, and the mischarging with histidine thus appears not to be of primordial biological importance. The different behaviours of the yeast and wheat germ HisRS enzymes also suggest that they utilize different minor identity determinants in selecting their RNA substrates.

\section{Recognition by elongation factors}

Elongation factors sequester aminoacylated tRNAs and carry them to the ribosome where the adaptor function of tRNA occurs (reviewed by Riis et al [59]). The aminoacylated TYMV tRNA-like structure, like charged tRNAs, interacts with elongation factors EFTu from $E$ coli [12], from Thermus thermophilus [43], and EF $1 \alpha$ from higher organisms [13]. The minimal sequence required for interaction with the $E$ coli factor was determined by retention experiments testing the binding of aminoacylated fragments to immobilized elongation factor [43]. A fragment of 47 nucleotides, corresponding to the 3 '-end of the viral RNA was the shortest RNA segment able to bind to the elongation factor. This fragment corresponds to the acceptor arm of the tRNA-like fragment, suggesting that the pseudoknot does not interfere with the binding events.

\section{Biology of the TYMV tRNA-like structure}

\section{Role in protein synthesis or in viral genome replication?}

Soon after the discovery of the efficient aminoacylation of plant viral genomes such as TYMV RNA, enquiries on the role of tRNA mimicry in the viral life cycle focussed on interactions with the ribosomes. It was thought that if interaction of charged viral RNA with the A site occurs in much the same way as that of tRNA ternary complexes, preferential translation of viral RNA may result. This might occur by displacement of host mRNAs, with or without actual donation of the amino acid charged on the viral RNA to the nascent polypeptide. However, no convincing evidence was ever obtained for amino acid donation [16]. Similar conclusions arose from studies on other aminoacylatable viral genomes $[60,61]$.

Subsequently, the focus has shifted towards an involvement of the tRNA-like structure directly in viral RNA replication. This was prompted by the expectation that RNA promoter sequences directing (-) strand synthesis are likely to be located near the 3'end of the genomic RNA, and by the adoption of the replicase of the RNA bacteriophage $Q \beta$ as a paradigm for the replicases of the plant viruses with aminoacylatable RNAs [62]. Core $Q \beta$ replicase is comprised of a virus-encoded polymerase subunit and three host subunits, EF-Tu, EF-Ts and the ribosomal protein S1

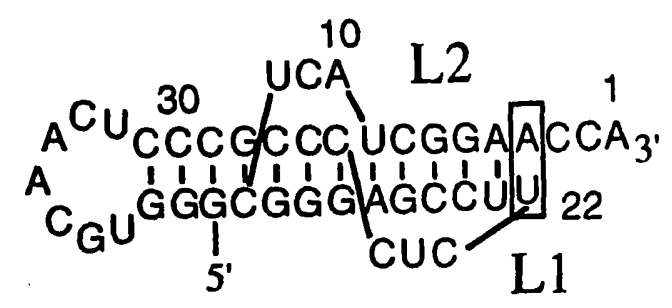

Fig 3. Minihelix mimicking the amino acid acceptor arm of the TYMV tRNA-like structure, with residue U22 opposite to discriminator base A4 (for numbering see legend to figure 1). 
[63]. It was suggested that the plant viral tRNA-like structures could sequester a similar selection of host proteins into the viral replication complex [62]. This postulate remains to be proven or disproven.

\section{Possible involvement of tRNA mimicry in TYMV RNA replication and role of aminoacylation}

It has been shown that the TYMV tRNA-like structure can be valylated in vivo, either in Xenopus laevis oocytes [64] or in Chinese cabbage leaves during a natural infection [65]. In Xenopus oocytes, the genomic RNA became fragmented, and the valylated species was a 4-5S RNA fragment of the genome [64]. The form of TYMV RNA valylated in Chinese cabbage plants was not determined, but since 3'-fragments and the entire genomic RNA can be valylated in vitro with similar efficiencies [24], it is assumed that at least some of the genomic RNAs are valylated during infection. The genomic and subgenomic RNAs in the virion lack the $3^{\prime}-\mathrm{A}$, and terminate in $3^{\prime}-\mathrm{CC}_{\mathrm{OH}}$ $[18,19]$, and so the encapsidated RNAs cannot be directly valylated. This implies that most of the newly synthesized viral RNAs - those destined for encapsidation - do not become valylated. Valylation is thus restricted to those viral RNAs that are engaged in translation or those that are participating in replication as templates. No way has yet been devised to directly assess the aminoacylation status of the viral RNAs engaged in these different functions.

The relevance of valylation to TYMV replication has been studied genetically using TYMV RNAs with mutations in the valine identity elements of the anticodon region. The same mutations used in TY-Smaderived RNAs to map the valine identity determinants [49] were transferred into genome-length transcripts, derivatives of the infectious TYMC RNAs made by transcription with T7 RNA rolymerase from cloned DNA from the TYMV Corvallis strain [8]. A direct correlation was observed between the replication of the genomic transcripts in Chinese cabbage protoplasts and the valylation efficiencies of the related 3'fragments as charged by wheat germ ValRS in vitro [66] (table I). A low basal level of RNA replication, detected as (-) strand synthesis in inoculated protoplasts, was observed for mutants with $V_{\text {max }} / K_{\mathrm{m}}$ values for in vitro valylation that were $<10^{-2}$ relative to wildtype (mutants with substitutions at the middle or 3'positions of the anticodon). These mutants were unable to infect whole plants. By contrast, mutants such as those with substitutions in the wobble position $(-57)$, which could be efficiently valylated, replicated to levels similar to wild type in both protoplasts and whole plants. Mutants with intermediate levels of valylatability (C-54 and A-53 mutants) replicated to intermediate levels in vivo (table I).
The correlation between replication and valylatability was further strengthened by the characterization of a mutant that arose spontaneously in a plant inoculated with TYMC-(A-55) RNA, which does not support systematic infection in plants. Apparently as a result of polymerase error during replication, the plant became systematically infected with progeny having a U-57/A-55 genotype. This genotype was reconstructed in TYMC-(U-57/A-55) RNA, which was shown to replicate to levels comparable to TYMC in vivo [52]. Besides replicating efficiently, this mutant RNA could also be relatively efficiently valylated: the RNA could be valylated to completion, and 3'-transcripts with the U-57/A-55 mutations were valylated with a $V_{\max } / K_{\mathrm{m}}$ value 0.075 relative to wild-type. The C-57U mutation has thus clearly suppressed the effect of the C-55A mutation in both the valylation and replication properties. The above data imply that valylation of the viral RNA by host ValRS is a requisite for the efficient replication of TYMV RNA.

The mechanistic basis of the requirement for valylation is not known at present, nor is it known whether efficient charging with an amino acid other than valine is compatible with efficient replication. It is likely that the valylation status of a molecule of TYMV RNA influences its potential as a template for (-) strand synthesis, the first stage of viral RNA replication, the effect being exerted at the level of $(-)$ strand promoter selection. It will be important to identify the host subunits of TYMV replicase, and so determine the validity of the $Q \beta$ replicase as a paradigm. Elongation factor EFI $\alpha$ would be a logically expected subunit, compatible with the correlation discussed above, but two studies have failed to detect this protein in preparations of TYMV replicase $[13,67]$.

\section{The role of a pseudoknot upstream of the tRNA-like structure}

Nucleotides between the CP-ORF termination codon and the tRNA-like structure can be folded into a pseudoknot (see fig 1), a structural element commonly found in viral RNAs and which has also been implicated in translational frameshifting in RNA viruses [68]. Because of their distinctive structure, pseudoknots may function as protein recognition elements involved in the regulation of translation and/or RNA replication.

In the course of studies on the role of the 3'-noncoding region of TYMV RNA, it was observed that mutation of U-96G, which destabilizes the basepairing needed for the formation of one of the helical segments of the pseudoknot upstream of the TYMV tRNA-like structure, resulted in a poor replication rate and inability to infect plants systematically [52]. When protoplasts infected with TYMC-(G-96) were 
used to inoculate Chinese cabbage plants, some plants did develop symptoms, but these infections were all due to progeny with altered sequences. Most plants contained progeny with simple G-96U reversions, but in a few cases, second-site suppressing mutations were responsible for the ability to develop systemic symptoms. In one such case, the suppressing mutation was found to be the A-107C substitution, which recovered the ability to form a pseudoknot similar to that present in the wild-type RNA (see fig 1) [52]. This mutation also abolished the normal CP-ORF termination codon, such that the extended CP-ORF terminated five codons downstream. TYMC-(C-107/G-96) was able to produce normal systemic symptoms in plants, but the virions were less stable than normal, resulting in the degradation of virion RNA in mature, infected leaves as virions dissociate. This suggests that the extended coat protein plays no role in rescuing the poor replication caused by the G-96 mutation, and that the rescue is due to the fact that the U-107C mutation recovers the ability to form a pseudoknot. The pseudoknot may represent a binding element recognized by the replication machinery in assembling a $(-)$ strand replication complex. It is interesting that the mutated nucleotides are protected when yeast ValRS binds TYMV RNA [31], but no altered valylation properties were observed for 3'transcripts with the G-96 mutation [52]. The presence of one or several pseudoknots in the 3'-non-coding region just dow'nstream of the stop codon is a common feature of plant viral RNA [69]. In the case of TMV RNA, these pseudoknots were shown to be necessary for the replication of the viral RNA [70].

\section{Possible role of the TYMV tRNA-like structure as the 3'telomere}

As mentioned above, TYMV virion RNA lacks the complete 3'-CCA OH required for aminoacylation, yet viral RNAs are known to be valylated in vivo. It is presumed that host tRNA nucleotidyl-transferase functions to add the missing 3'-A residue to disencapsidating RNAs after inoculation. tRNA nucleotidyltransferase serves to maintain intact 3'-termini on cytoplasmic tRNA molecules, whose 3 '-ends are constantly at risk from exonuclease attack and which turn rapidly over in the cell [42]. The site of (-) strand initiation on the tRNA-like structure template has not been determined for TYMV, but it is likely to be within one or two nucleotides of the 3'-end, as has been shown with BMV [71]. Thus, loss of 3-nucleotides could cause the loss of the $(-)$ strand initiation site and abortion of the entire replication cycle. Thus, the role of host tRNA nucleotidyl-transferase in maintaining intact 3'-ends is probably a crucial one, and is a role analogous to that of the telomerase enzymes that add T/G-rich sequences to the ends of linear DNA chromosomes to prevent the loss of terminal sequences during replication [72]. Since the tRNAlike structure is a feature that makes the viral RNA a substrate for tRNA nucleotidyl-transferase, the tRNAlike structure may be considered the telomere of the linear viral RNA genome. While this functional role is supported by experimental evidence in the case of BMV RNA [73], it remains to be demonstrated for TYMV RNA.

\section{Function of the tRNA-like structure as promoter for (-) strand synthesis}

Studies with TYMV are not yet sufficiently advanced to provide as convincing a case as with $\mathrm{BMV}$ for the involvement of the tRNA-like structure in promoting $(-)$ strand synthesis. Nevertheless, data consistent with such a role for the TYMV tRNA-like structure exist. Morch et al [74] used short RNA transcripts from the 3'-end of the genome as templates in vitro for a TYMV replicase preparation, and as inhibitors of the copying of TYMV RNA. Despite the presence of a 3'-tract of 50 adenosine residues and 31 further heterologous nucleotides, an RNA containing the 100 3'-nucleotides of TYMV RNA (ie including the tRNA-like structure) was able to function as a template for RNA synthesis [74]. This RNA was also able to inhibit the copying of TYMV RNA when present at 2.5- to 25-fold molar excess. A related RNA containing a longer segment from the 3'-end of TYMV RNA had similar properties, but these were abolished by the removal of the tRNA-like structure. In further studies, shorter fragments containing only part of the tRNA-like structure were tested in the above assays. RNAs containing as few as 38 nucleotides from the 3'-end of TYMV RNA (ic the amino acid acceptor stem and part of the T-stem analogue) as well as eight unspecified nucleotides at the 5 '-end and 13 at the 3'-end, were able to inhibit TYMV RNA copying in vitro, and appeared to act as templates for the TYMV replicase.

In preliminary experiments using a TYMV replicase preparation, we have obtained results consistent with the above observations (Georgel and Dreher, unpublished). Transcripts with correct 3'-CCA termini and containing variable lengths of sequence from the 3'-end of TYMV RNA were used as templates for TYMV replicase. RNAs derived from TY-Sma, TYDde, TY-Dra and TY-Alu clones and containing 258, 109, 93 and 82 nucleotides of TYMV sequence were all active templates. Even TY- $A A$, a 42-nucleotidelong RNA representing the 3'-half of the tRNA-like structure with a slightly reconstructed pseudoknot (see fig 3), was able to serve as a template. The copying of these molecules was inhibited by a derivative of TY- 

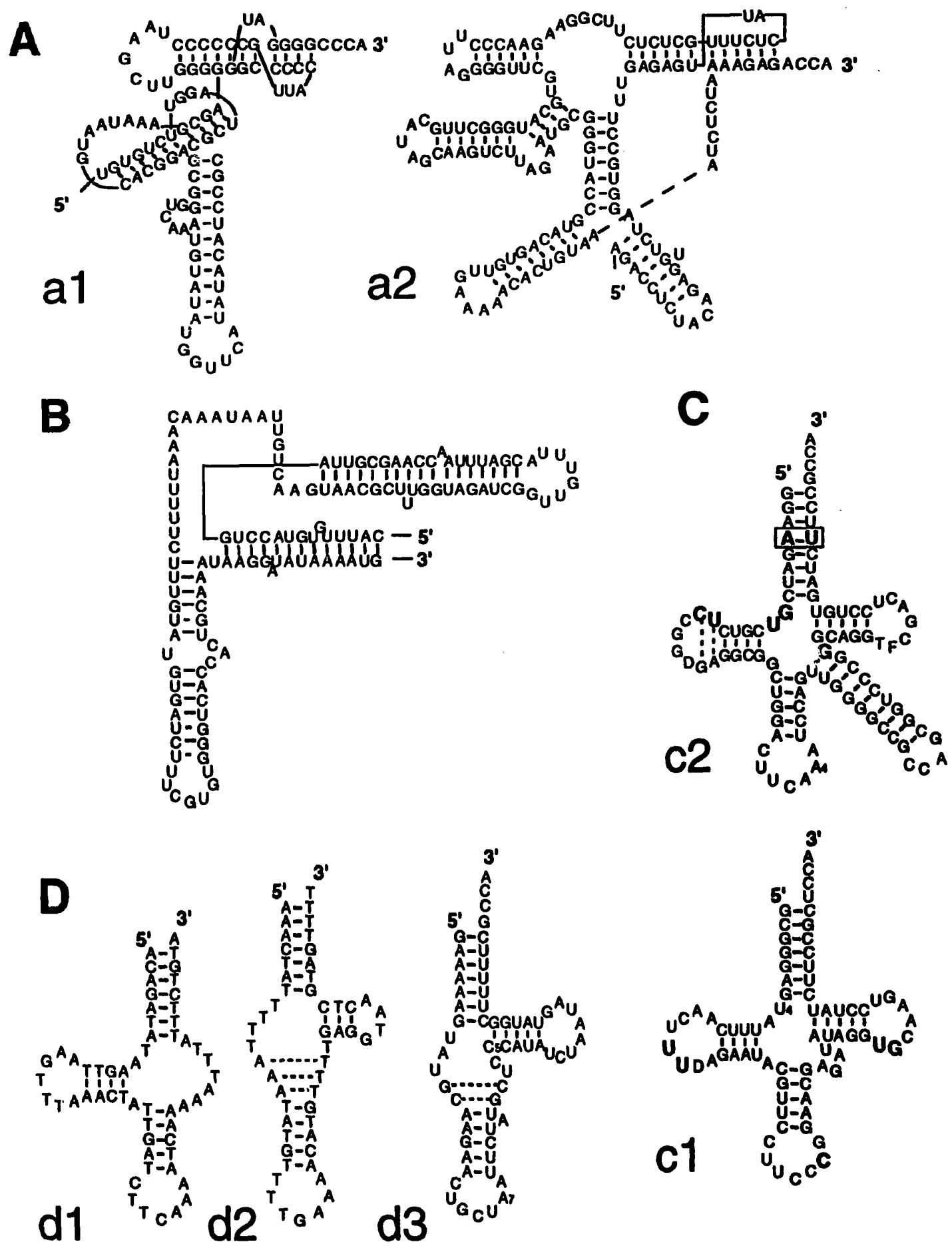

Fig 4. Other tRNA mimicries. A. Plant viral tRNA-like domains from TMV (a1) and BMV (a2). B. tRNA-like region with the regulatory negion of the messenger RNA of $E$ coli ThrRS. C. tRNAs with minor structural changes: (c1) tRNAGly from $S$ epidermidis and (c2) tRNASec (specific for selenocysteine) from $E$ coli. D. Bizarre mitochondrial tRNA sequences from nematodes (obtained from gene sequencing) ( $\mathrm{d} 1$ and $\mathrm{d} 2$ ) and from bovine heart (d3). In . C, structural features in tRNA sequences deviating from standards are shown. 
Sma containing 18 additional 3'-nucleotides (GGGGUACCGAGCUCGAAU), an RNA that was itself not copied by the TYMV replicase (Georgel and Dreher, unpublished).

The above results thus are consistent with the localization of the (-) strand promoter to the 3'-tRNA-like structure of TYMV RNA. We remain concerned, however, about the specificity of the in vitro studied TYMV replicase, and the relevance of the results obtained with TYMV infection processes.

\section{Other functional possibilities}

Besides the replication-related roles discussed above, it is likely that other, perhaps more subtle roles for the tRNA-like structure will emerge in the future. It has been suggested that the sequences forming the TYMV tRNA-like structure may assume an alternative conformation involving base-pairing with 5'-terminal sequences [75]. Such pairing is suggested by lightscattering experiments [76], and would prevent recognition of both the initiation codons of ORF-69 and ORF-206, and of the tRNA-like structure. This may play a role in the regulation of the infection process. The 3'-non-coding regions of viral RNAs (especially TMV RNA) have been shown to increase mRNA expression in a number of cell types $[77,78]$, and may thus be important in ensuring adequate viral gene expression. Other regulatory roles involving RNA binding to the ribosome along the lines of the suggestions discussed earlier should not be disregarded. Ribosomes are known to play a role in the active disencapsidation of viruses $[79,80]$, and it would be interesting to determine with modern binding studies whether the tRNA-like structure has any affinity for ribosomes.

\section{Other tRNA-like molecules and mimicries with tRNAs}

The presence of a tRNA-like domain within the RNA for TYMV is not an exception in the constitution of viral genomes. Several other plant viruses present similar functional domains at the 3'-end of their RNA genomes. Besides tymoviruses, whose RNAs specifically charge valine, there are two other large groups of viruses presenting an aminoacylatable domain. Tobamoviruses are histidinylatable (with one exception, the CcMV strain, charged with valine) and bromo-, cucumo-, and hordeiviruses are all tyrosylatable. All these viral RNAs are also recognized by a series of tRNA-specific proteins like elongation factors and tRNA nucleotidyl-transferases (reviewed by Mans et al [50] and Florentz and Giegé [81]). Their structural mimicry with tRNAs is much more complicated than that found for TYMV (fig 4A). The extreme case is RNA from BMV that presents the most intricate sequence (fig 4a2), and nevertheless is well charged by TyrRS. As for the TYMV tRNA-like domain, the biological role of these structures is now being deciphered $[50,81]$, and involvement during replication is also strongly suggested in the case of BMV. But in contrast to TYMV RNA, aminoacylation is not required for BMV RNA replication $[71,82]$.

tRNA mimicries have also been found in several other biological systems. Thus, in messenger RNAs coding for aminoacyl-tRNA synthetases ( $E$ coli HisRS, ThrRS and MetRS), the regulatory region of the mRNA contains tRNA-like elements [83-85]. For ThrRS mRNA, the structure of the tRNA-like domain is well established (fig 4B) [86] and it was shown that interaction with the synthetase regulates the level of transcription of the gene $[86,87]$. Interestingly, even for natural tRNAs, a large variability in structure has been revealed (fig 4C, D). Small sequence variations have been found in tRNAs involved in specialized biological functions. For example, tRNAGly from Staphylococcus epidermidis, which participates in cell wall synthesis (but not in classical protein synthesis)

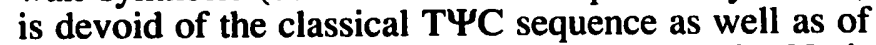
canonical D-loop characteristics (fig 4c1) [88]. Noticeable are the sequence similarities between this glycine-specific tRNA and the homologous regions in the TYMV tRNA-like domain (see fig 1) [25], which also is not involved in ribosome directed protein synthesis. The recently discovered tRNA ${ }^{\text {Sec }}$ species, incorporating the 21 th amino acid selenocysteine into a limited number of proteins has an acceptor arm with 13 base-pairs and presents peculiar tertiary interactions $[89,90]$ (see fig 4c2 for the secondary fold of $E$ coli tRNASec). Large structural changes can also occur in other tRNAs participating in protein synthesis. This was first found in mammalian mitochondrial tRNASer species lacking the entire D-loop and stem $[91,92]$ (fig 4D). More recently a series of other mitochondrial tRNAs have been discovered with bizarre structures, in particular in nematodes (fig 4D) [93], in which D-stems, T-stems, or both of them show strong structural deviations and can even be absent.

What is the evolutionary significance of the great structural variability within RNA molecules that can be recognized by tRNA specific proteins? While a common origin can be postulated for $t R N A s$ presenting a cloverleaf structure (even if parts of the cloverleaf are missing), there are strong theoretical arguments for a different evolutionary origin of tRNA-like molecules in which the tRNA mimicry results from different architectural principles (as in plant viral RNAs where pseudoknots are required). Thus, it is likely that molecular tinkering events occurred in the early development of life when strategies for both 
transcriptional and translational mechanisms had to be found by nature. Because these processes are so basic in all forms of life, they could have involved common molecules, but also alternate structures able to fulfil similar functions. This scenario is not unlikely, since in vitro or in vivo genetic selection methods have recently generated RNA molecules with functional tRNA characteristics but presenting novel structural features not found in known tRNAs (or tRNA-like molecules) $[94,95]$.

As a conclusion, and from a more practical point of view, we emphasize that tRNA-like molecules are excellent tools to understand tRNAs. Studies on TYMV RNA valylation have shown that the functional strategy used by nature to ensure the aminoacylation identity of this RNA is the same as for canonical tRNAs. Indeed, the identity nucleotides responsible for valylation are located at topologically similar places in the structure of both types of molecules [48, 49, 81]. Extending this concept, it should be possible to engineer molecular substrates of synthetases in which identity elements are carried by adequate structural frames allowing them to interact with their counterparts on the synthetases. A first application came from the engineering of an RNA minihelix derived from the TYMV tRNA-like domain, in which the histidine identity element is mimicked by a residue from the terminal pseudoknot that structurally mimicks the major identity nucleotide from histidine specific IRNAs (see above). As anticipated, the minihelix became an efficient substrate for HisRS [55].

\section{Acknowledgment}

We dedicate this paper to the memory of Professor Léon Hirth (1920-1991). He contributed enormously to the development of plant virology in France and promoted studies on TYMV RNA. He encouraged our projects on the aminoacylation of TYMV RNA at a time when this phenomenon was not well understood. We acknowledge the many discussions we had with him on this topic and on its relevance to virology.

\section{References}

1 Markhum R, Smith KM (1949) Studies on the virus of turnip yellow mosaic. Parasitology 39, 330-342

2 Hirth L. Givord L (1988) Tyınoviruses. In: The plant viruses (Koenig R, ed) Plenurn Publishing Corporation, vol 3, 163-212

3 Morch MD. Boyer JC, Haenni AL (1988) Overlapping open reading frames revealed by nucleotide sequencing of turnip yellow mosaic virus genomic RNA. Nucleic Acidis Res 16, 6157-6173

4 Dreher TC, Bransom KL (1992) ( enomic RNA sequence of turnip yellow mosaic virus is late TYMC, a cDNAbased clone with verified infectivity. Plant Mol Biol 18, 403-406
5 Keese P, Mackenzie A, Gibbs A (1989) Nucleotide sequence of the genome of an Australian isolate of turnip yellow mosaic tymovirus. Virology 172, 536-546

6 Morch MD, Drugeon G, Szafranski P, Haenni AL (1989) Proteolytic origin of the 150 -kilodalton protein encoded by turnip yellow mosaic virus genomic RNA. J Virol 63, 5153-5158

7 Bransom KL, Weiland JJ, Dreher TW (1991) Proteolytic maturation of the 206-kDa nonstructural protein encoded by turnip yellow mosaic virus RNA. Virology 184, 351-358

8 Weiland JJ, Dreher TC (1989) Infectious TYMV RNA from cloned cDNA. Effects in vitro and in vivo of point substitutions in the initiation codons of two extensively overlapping ORFs. Nucleic Acids Res 17, 4675-4687

9 Beljanski M (1965) L'ARN isolé du virus de la mosaïque jaune du navet accepteur des L-acides-aminés en présence d'enzymes bactériens. Bull Soc Chim Biol 47, 1645-1652

10 Pinck M, Yot P, Chapevillc F, Duranton H (1970) Enzymatic binding of valint to the 3'-end of TYMV RNA. Nature 226, 954-956

11 Yot P, Pinck M, Haenni AL, Duranton H, Chapeville F (1970) Valine-specific tRNA-like structure in turnip yellow mosaic virus RNA. Proc Natl Acad Sci USA 67, 1345-1352

12 Litvak S, Tarrago A, Tarrago-Litvak L, Allende JE (1973) Host elongation factor in vitro interaction with TYMV and TMV genome depends on viral tRNA aminoacylation. Nature New' Biol 241, 88-93

13 Joshi RL, Ravel JM, Haenni AL (1986) Interaction of turnip yellow mosaic virus Val-RNA with eukaryotic elongation factor EF-1 $\alpha$. Search for a function. $E M B O J 5$, $1143-1148$

14 Litvak S, Tarrago-Litvak L, Chapeville F (1973) TYMVRNA as a substrate of transfer RNA nucleotidyl-transferase. II. Incorporation of cytidine 5'-monophosphate and determination of a short nucleotides sequence at the 3'-end of the RNA. I Virology 11, 238-42

15 Prochiantz A, Haenni AL (1973) TYMV RNA as a substrate of the tRNA maturation endonuclease. Nature 241, $168-170$

16 Haenni AL, Joshi S, Chapeville F (1982) tRNA-like structures in the genomes of RNA viruses. Prog Nucleic Acid Res Mol Biol 27, 85-104

17 Giegé R, Briand JP, Mengual R, Ebel JP, Hirth L (1978) Valylation of the two RNA components of turnip yellow mosaic virus and specificity of the aminoacylation reaction. Eur J Biochem 84, 251-256

18 Briand JP, Jonard G, Guilley $H$, Richards $K$, Hirth L (1977) Nucleotide sequence $(n=159)$ of the amino-acidaccepting 3'-OH extremity of turnip-yellow-mosaic-virus RNA and the last portion of its coat-protein cistron. Eur $J$ Biochem 72, 453-463

19 Silberklang M, Prochiantz A, Haenni AL, RajBhandary UL (1977) Studies on the sequence of the $3^{\prime}$-terminal region of turnip yellow mosaic virus RNA. Eur J Biochem $72,465-478$

20 Pleij CWA, Rietveld K, Bosch L (1985) A new principle of folding based on pseudoknotting. Nucleic Acids Res 13, 1717-1731

21 Pleij CWA (1990) Pseudoknots: a new motif in the RNA game. Trends Biochem Sci 15, 143-147

22 ten Dam E, Pleij K, Draper D (1992) Structural and functional aspects of RNA pseudoknots. Biochemistry 31, 11665-11676

23 Westhof E, Jaeger L (1992) RNA pseudoknots: structural and functional aspects. Curr Opinion Struct Biol 2, 327-333 
24 Florentz C, Mengual R, Briand JP, Giegé R (1982) Largescale purification of the 3'-OH-terminal tRNA-like sequence $(n=159)$ of turnip yellow mosaic virus RNA. Eur J Biochem 123, 89-93

25 Florentz C, Briand JP, Romby P, Hirth L, Ebel JP, Giegé R (1982) The tRNA-like structure of turnip yellow mosaic virus RNA: structural organization of the last 159 nucleotides from the 3'-OH terminus. EMBO J 1, 269-276

26 Rietveld K, Van Poelgeest R, Pleij CWA, Van Boom JH, Bosch L (1982) The tRNA-like structure at the 3'-terminus of turnip yellow mosaic virus RNA. Differences and similarities with canonical tRNA. Nucleic Acids Res 10, 19291946

27 Dumas P, Moras D, Florentz C, Giegé R, Verlaan P, Van Belkum A, Pleij CWA (1987) 3-D graphics modelling of the tRNA-like $3^{\prime}$-end of turnip yellow mosaic virus RNA: structural and functional implications. $J$ Biomol Struct Dyn 4, 707-728

28 Van Belkum A, Bingkun J, Rietveld K, Pleij CWA, Bosch L (1987) Structural similarities among valineaccepting tRNA-like structures in tymoviral RNAs and elongator tRNAs. Biochemistry 26, 1144-1151

29 Mans RMW, Van Steeg MH, Verlaan PWG, Pleij CWA, Bosch L (1992) Mutational analysis of the pseudoknot in the tRNA-like structure of turnip yellow mosaic virus RNA. J Mol Biol 223, 221-232

30 Rietveld K (1984) Three-dimensional folding of the tRNA-like structures of some plant viral RNAs. Thesis, Leiden, the Netherlands

31 Florentz C, Giegé R (1986) Contact areas of the turnip yellow mosaic virus tRNA-like structure interacting with yeast valyl-tRNA synthetase. $J$ Mol Biol 191, 117-130

32 Westhof E, Romby P, Ehresmann C, Ehresmann B (1990) Computer-aided structural biochemistry of ribonucleic acids. In: Theoretical biochemistry and molecular biophysics (Beveridge D, Lavery R, eds) Adenine Press, Guilderland, NY, USA, 399-409

33 Dock AC, Lorber B, Moras D, Pixa G, Thierry JC, Giegé R (1984) Crystallization of transfer ribonucleic acids. Biochimic 66, 179-201

34 Ducruix A, Giegé R (eds) (1992) Crystallization of nucleic acids and proteins: $A$ practical approach. The practical approach series (Rickwood D, Hames BD, eds) IRL Press at Oxford Uniiversity. Press, Oxford, 331

35 Westhof E, Dumas P, Moras D (1985) Crystallographic refinement of yeast aspartic acid transfer RNA. I Mol Biol $184,119-145$

36 Giegé R, Rudinger J, Dreher T, Perret V, Westhof E, Florentz C, Ebel JP (1990) Search of essential parameters for the aminoacylation of viral tRNA-like molecules. Comparison with canonical transfer RNAs. Biochem Biophys Acta 1050, 179-185

37 Altman S (1990) Ribonuclease P. J Biol Chem 265, 20053-20056

38 Brown JW, Pace NR (1991) Structure and evolution of ribonuclease P RNA. Biochimie 73, 689-697

39 Guerrier-Takada C, Van Belkum A, Pleij CWA, Altman S (1988) Novel reactions of RNAase $P$ with a tRNA-like structure in turnip yellow mosaic virus RNA. Cell 53, 267-272

40 Green CJ, Vols BS, Morch MD, Joshi RL, Haenni AL (1988) Ionic condition for the cleavage of the tRNA-like structure of turnip yellow mosaic virus by the catalytic RNA of RNAse P. J Biol Chem 263, 11617-11620

41 Mans RMW, Guerrier-Takada C, Aliman S, Pleij CWA (1990) Interaction of RNAse P from Escherichia coli with pseudoknotted structures in viral RNAs. Nucleic Acids Res 18, 3479-3487

42 Deutscher MP (1983) tRNA nucleotidyl-transferase and the CCA terminus of transfer RNA. In: Enzymes of nucleic acid synthesis and modification, vol 2 (Jacob ST, ed) CRC Press, Inc, Boca Raton, FL, USA, 159-183

43 Joshi S, Chapeville F, Haenni AL (1982) Length requirements for tRNA specific enzymes and cleavage specificity at the 3'-end of turnip yellow mosaic virus RNA. Nucleic Acids Res 10, 1947-1962

44 Kern D, Giegé R, Ebel JP (1972) Incorrect aminoacylations eatalysed by the phenylalanyl- and valyl-tRNA synthetase from yeast. Eur J Biochem 31,148-155

45 Dreher TW, Florentz C, Giegé R (1988) Valylation of tRNA-like transcripts from cloned cDNA of turnip yellow mosaic virus RNA demonstrate that the L-shaped region at the 3'-end of the viral RNA is not sufficient for optimal aminoacylation. Biochimie 70, 1719-1727

46 Mans RMW, Verlaan PWG, Pleij CWA, Bosch L (1990) Aminoacylation of 3'-terminal tRNA-like fragments of turnip yellow mosaic virus RNA: the influence of 5'nonviral sequences. Biochem Biophys Acta 1050, 186192

47 Favorova OO, Fasiolo F, Keith G, Vassilenko SK, Ebel JP (1981) Partial digestion of tRNA-aminoacyl-tRNA synthetase complexes with cobra venom ribonuclease. Biochemistry 20, 1006-1010

48 Florentz C, Dreher TW, Rudinger J, Giegé R (1991) Specific valylation identity of turnip yellow mosaic virus RNA by yeast valyl-tRNA synthetase is directed by the anticodon in a kinetic rather than affinity-based discrimination. Eur J Biochem 195, 229-234

49 Dreher TW, Tsai C-H, Florentz C, Giegé R (1992) Specific valylation of turnip yellow mosaic virus RNA by wheat germ valyl-tRNA synthetase is determined by three anticodon loop nucleotides. Biochemistry 31, 9183-9189

50 Mans RMW, Pleij CWA, Bosch L (1991) tRNA-like structures. Structure, function and evolutionary signiticance. Eur.J Biochem 201, 303-324

51 Fersht A (1985) Enzyme, structure and mechan'sm. Freeman, New York, USA, 371

52 Tsai CH, Dreher TW (1992) Second-site suppressor mutations assist in studying the function of the $3^{3}$-noncoding region of turnip yellow mosaic virus RNA. I Virolog.' 66 , 5190-5199

53 Ebel JP, Giegé R, Bonnet J, Kern D, Befort N, Bollack C. Fasiolo F, Gangloff J, Dirheimer G (1973) Factors determining the specificity of the TRNA aminoacylation reaction. Biochimie 55, 547-557

54 Giegé R, Puglisi JD, Florentz C (1993) tRNA structure and aminoacylation efficiency. Prog Nucleic Acid Res Mol Biol 45, 128-206

55 Rudinger J, Florentz C, Dreher T, Giegé R (1992) Efficient mischarging of a viral tRNA-like structure and aminoacy!ation of a minihelix containing a pseudoknot: histidinylation of turnip yellow mosaic virus RNA. Nucleic Acids Res 20, 1865-1870

56 Himeno $H$, Hasegawa T, Ueda T, Watanabe K. Miura K, Shimizu M (1989) Role of the extra G-C pair at the end of the acceptor stem of tRNA ${ }^{\text {His }}$ in aminoacylation. Nucleic Acids Res 17, 7855-7863

57 Francklyn C, Schimmel P (1990) Enzymatic aminoacylation of an eight-base-pair microhelix with histidine. Proc Natl Acad Sci USA 87, 8655-8659

58 Francklyn C, Musier-Forsyth K, Schimmel P (1992) Small RNA helices as substrates for aminoacylation and their 
relationship to charging of transfer RNAs. Eur $J$ Biochem 206, 315-321

59 Riis B, Rattan SIS, Clark BFC, Merrick WC (1990) Eukaryotic protein elongation factors. Trends Biochem Sii 15 , 420-424

60 Chen JM, Hall TC (1973) Comparison of tyrosyl transfer ribonucleic acid and brome mosaic virus tyrosyl ribonucleic acid as amino acid donors in protein synthesis. Biochemistry 12, 4570-4574

61 Hall TC, Pinck M, Duranton HM, German TL (1979) Aminoacylation and messenger functions of eggplant mosaic virus RNA. Virology 97, 354-365

62 Hall TC (1979) Transfer RNA-like structures in viral genomes. Int Rev Cytol 60, 1-26

63 Blumenthal T, Carmichael GG (1979) RNA replication: function and structure of $\Omega \beta$-replicase. Annu Rev Biochem 48, 525-548

64 Joshi S. Haenni AL, Hubert E, Huez G, Marbaix G (1978) In vivo aminoacylation and 'processing' of turnip yellow mosaic virus RNA in Xenopus laevis oocytes. Nature 275, 339-341

65 Joshi S, Chapeville F, Haenni AL (1982) Turnip yellow mosaic virus RNA is aminoacylated in vivo in Chinese cabbage leaves. EMBO J 1, 935-938

66 Tsai CH, Dreher TW (1991) Turnip yellow mosaic virus RNAs with anticodon loop substitutions that result in decreased valylation fail to replicate efficiently. $J$ Virology $65,3060-3067$

67 Pulikowska J, Wojtaszek P, Korez A, Michalski Z, Candresse T, Twaerdowski T (1988) Immunochemical properties of elongation factors 1 of plant origin. Eur.$J$ Biochem $171,131-136$

68 Brierley I, Digard P. Inglis SC (1989) Characterization of an efficient coronavirus ribosomal frameshifting signal: requirement for an RNA pseudoknot. Cell 57 , $537-547$

69 Pleij CWA. Abrahams JP. Van Belkum A, Rietveld K, Bosch $L$ (1987) The spatial folding of the 3 ' noncoding region of aminoacylatable plant viral RNAs. III: Positiv' Sirand RNA Viruses (Brinton MA, Rueckert $R$, eds) UCLA Symposia on Molecular and Cellular Biology, New Series 54, 299-316

70 Takamatsu NY, Watanabe Y, Meshi T, Okada Y (1990) Mutational analysis of the pseudoknot region in the $3^{\prime}$ noncoding region of tobacco mosaic virus RNA. $J$ Gen Virol 64, 3686-3693

71 Miller WA, Bujarski JJ, Dreher TW, Hall TC (1986) Minus-strand initiation by brome mosic virus replicas. within the $3^{\prime}$ tRNA-like structure of native and modified RNA templates. I Mol Biol 187, 537-546

72 Blackburn EH (1992) Telomerases. Anmu Rev Biochem 61, 113-129

73 Rao ALN, Dreher TW, Marsh LE, Hall TC (1989) Telomeric function of the tRNA-like structure of brome niosaic virus RNA. Proc Natl Acad Sci USA 86, 5335-5339

74 Morch MD, Joshi RL, Denial TM, Haenni AL (1987) A new 'sense' RNA approach to block viral replication in vitro. Nucleic Acids Res 15, 4123-4130

75 Florentz C, Briand JP, Giegé R (1984) Possible functional role of viral tRNA-like structures. FEBS Lett 176, 295300

76 Strazielle C, Benoit H, Hirth L (1965) Particularités structurales de l'acide nucléique extrait du virus de la mosaïque jaune du navet. J Mol Biol 13,735-748

77 Gallie DR, Walbot V (1990) RNA pseudoknot domain of tobacco mosaic virus can functionally substitute for a
poly(A) tail in plant and animal cells. Genes Dev 4, 1149-1157

78 Gallie DR, Feder JN, Schimke RT, Walbot V (1991) Functional analysis of the tobacco mosaic virus tRNA-like structure in cytoplasmic gene regulation. Nucleic Acids Res $19,5031-5036$

79 Wilson TMA (1984) Cotranslational disassembly of tobacco mosaic virus in vitro. Virology 137, 255-265

80 Singh I, Helenius A (1992) Nucleocapsid uncoating during entry of enveloped animal RNA viruses into cells. Sem Virol 3, 511-518

81 Florentz C, Giegé R (1993) tRNA-like structures in viral RNAs. In: Transfer RNA (Söll D, RajBhandary UL, eds) American Society Microbiology, in press

82 Dreher TW, Hall TC (1988) Mutational analysis of the sequence and structural requirements in brome mosaic virus RNA for minus strand promoter activity. $J$ Mol Biol $201,31-40$

83 Ames BN, Tsang TH, Buck M, Christman MF (1983) The leader mRNA of the histidine attenuator region resembles tRNAHis; possible general regulatory implications. Proc Natl Acad Sci USA 80, 5240-5242

84 Springer M, Graffe M, Butler JS, Grunberg-Manago M (1986) Genetic definition of the translational operator of the threonine tRNA ligase gene in Escherichia coli. Proc Natl Acad Sci USA 83, 4384-4388

85 Dardel F, Panvert M, Fayat G (1990) Transcription and regulation of expression of the Escherichia coli methionyl-tRNA synthetase gene. Mol Gen Genet 223, 121-133

86 Graffe M, Dondon J, Caillet J, Romby P, Ehresmann C, Ehresmann B, Springer M (1992) The speciticity of translational control switched using tRNA identity rules. Science 255, 994-996

87 Moine H, Romby P, Springer M, Grunberg-Manago M, Ebel JP, Ehresmann B, Ehresmann C (1990) Escherichia coli threonyl-tRNA synthetase and IRNAThr modulate the binding of the ribosome to the translational initiation site of the ihrS mRNA. I Mol Biol 216, 299-310

88 Roberts RJ (1974) Staphylococcal transfer ribonucleic acids. II. Sequence analysis of isoaccepting glycine transfer ribonucleic acids Ia and Ib from Staphylococcus epidermidis Texas 26. J Biol Chem 249, 4787-4796

89 Baron C, Westhof E, Böck A, Giegé R (1993) Solution structure of selenocysteine inserting tRNA Sec from Escherichia coli. Comparison with canonical tRNASer. J Mol Biol 231, 274-292

90 Sturchler C, Westhof, E, Carbon P, Krol A (1993) Unique secondary and tertiary structural features of the eucaryotic selenocysteine tRNA Sec. Nucleic Acids Res 21, 1073-1079

91 Arcari P, Brownlee GG (1980) The nucleotide sequence of a small (3S) seryl-tRNA (anticodon GCU) from beef heart mitochondria. Nucleic Acids Res 8, 5207-5212

92 de Bruijn MHL, Klug A (1983) A model for the tertiary structure of mammalian mitochondrial transfer RNAs lacking the entire 'dihydrouridine' loop and stem. EMBO J 2 , 1309-1321

93 Wolstenholme DR, Macfarlane JL, Okimoto R, Clary DO, Wahleithner JA (1987) Bizarre tRNAs inferred from DNA sequences of mitochondrial genomes of nematode worms. Proc Natl Acad Sci USA 84, 1324-1328

94 Hou YM, Schimmel P (1992) Novel transfer RNAs that are active in $E$ coli. Biochemistry $31,4157-4160$

95 Pan T, Uhlenbeck OC (1992) In vitro selection of RNAs that undergo autolytic cleavage with $\mathrm{Pb}^{2+}$. Biochemistry 31, 3887-3895 